\title{
The Importance of Importance
}

\section{Self-Descriptors in Dysphoria}

\author{
J.K. Brinker ${ }^{1}$, J. Aitken Harris², B. Guyitt ${ }^{3}$, and D.J.A. Dozois ${ }^{1}$ \\ ${ }^{1}$ Department of Psychology, University of Western Ontario, Social Science Center, London, Ontario, \\ ${ }^{2}$ Management and Organizational Studies, Faculty of Social Science, University of Western Ontario, \\ London, Ontario, ${ }^{3}$ Department of Psychology, University of New Brunswick, Fredericton, NB, all Canada
}

\begin{abstract}
This study examined the relationship between importance ratings of positive and negative personal attributes and depressed mood. Undergraduate psychology students $(n=115)$ completed the Beck Depression Inventory-II and made self-referential ratings on several adjectives. Participants subsequently indicated how important it was for them to possess or fail to exhibit each of these traits. The results demonstrated that the perceived lack of important positive traits was related to increased depressed mood whereas not exhibiting important negative traits was associated with less depressed mood. Moreover, depressed mood was related to the degree to which respondents were certain about their endorsement of the traits. The implications of these results are discussed.
\end{abstract}

Keywords: personal attributes, mood, self-concept

Recent advances in cognitive theories of depression have provided researchers with a much better understanding of how cognitive factors relate to mood. The concept of the self-schema was developed to help describe a mental structure that allows individuals to attend to and process information relating to the self more efficiently (Clark, Beck, \& Alford, 1999; Segal, 1988). Thus, information that is consistent with the self-schema is more likely to be attended to, and more easily processed, than information that is inconsistent with the self (Segal \& Gemar, 1997). Beck's (1967) model of depression suggests that people who experience depression possess a depressogenic self-schema consisting of negative information or beliefs about the self. The information in the depressogenic self-schema is vast and tightly interwoven allowing negative information to be selectively attended to and processed very efficiently (Ingram, 1984). By this mechanism, the depressive self-schema perpetuates itself and fosters further growth and organization of negative information. Individuals who do not experience depression are thought to lack this highly integrated network of negative information about the self.

It could, however, be argued that even the happiest and most self-satisfied person recognizes negative aspects of themselves. Is it simply the quantity of negative aspects of self that lead to dysphoria? Is it the closely interconnected structure of the negative self-schema that brings about depression? Another possibility may lie in the value that is placed on the individual aspects of self. If a person deems certain characteristics as unimportant to self-worth, lacking those qualities should not affect their mood. However, if the person deems these qualities as very important, realization of not possessing them could lead to depressed mood. Take the example of a person who is overweight and intelligent. If being thin is of utmost importance, this person may well experience depressed mood. However, if the person values intelligence over appearance, he or she may be able to overlook personal physical imperfections, comforted by the knowledge that she or he still possesses an important positive quality: intelligence. Past research has supported the relationship between the differential importance of personal qualities and self-esteem (Pelham, 1995b; Pelham \& Swann, 1989). This article examines the role that importance of the self descriptor plays in relation to depressed mood.

\section{Self-Schema Theory}

Schemas are internal networks of information, built from experience with the world, which allow the individual to process information about a given concept more efficiently (Clark et al., 1999; Segal, 1988). By possessing a structure of information, individuals are able to identify, encode, and categorize new information without expending considerable amounts of cognitive energy. The schema is not only a passive store of previously learned information, but also actively directs the individual's attention, perception, and encoding of new information (Gotlib \& Krasnoperova, 1998). Information that is congruent with the schema is more likely to be attended to and more efficiently processed than information that is incongruent with the schema. Thus, schemas produce an active bias toward information that is congruent with schema content.

This bias is particularly important for the idea of the depressive schema. Individuals who suffer from depression possess a negative view of themselves, their world, and their future (Beck, 1967). The accumulation of such cog- 
nitions, images, and attitudes form an organized network called a "depressive schema." The depressive schema is proposed to consist of highly organized negative information about the self. The content of the schema is stable and does not fluctuate with mood states. These schemas then affect the way in which information is processed and cause the individual to distort information in the environment leading to misinterpretation of events. As mentioned, schemas influence what new information is attended to, how the information is perceived, and what expectancies a person has regarding the information. Thus, individuals who possess a depressive self-schema are more likely to attend to and process negative information, because it is congruent with the content of their self-schema.

The content of the self-schema has generally been assessed through participants' endorsements of descriptive adjectives, and the recall of those adjectives. Using a semantic processing paradigm, Derry and Kuiper (1981) found that participants were more likely to recall self-referent words than words on which they had made structural or semantic ratings. Nonpsychiatric controls and nondepressed psychiatric controls showed enhanced recall only for self-referent nondepressed-content adjectives. Depressed participants, however, showed increased recall only for self-referent depressed-content adjectives. Such increased recall suggested that the information was processed in a schema-congruent fashion and provided evidence for a depressive schema. Further, measuring reaction times of endorsing the adjectives was used to assess certainty of endorsement. The authors proposed that when participants are able to rate items very quickly, they are more certain about their answers. When participants take more time to rate the items, they require the time to decide whether the adjective is self-descriptive or not.

\section{Differential Importance of Self-Descriptors}

Rosenberg (1979) suggested that esteem is not simply based on possessing certain qualities, "but on an assessment of the qualities that count (original italics)" (p. 18). William James (1890) proposed this idea in his early writings and explained that it is the personal characteristics that an individual places the utmost importance on that are crucial to self-esteem. The value an individual places on various attributes would remain stable over time. Rosenberg (1965) was the first to empirically test this theory and found that individuals who possessed negative attributes were more likely to experience low self-esteem if they felt these attributes were personally important. Subsequent research, however, was unable to replicate these findings, finding only weak support (Kaplan, 1980; Marsh, 1986). Some researchers even found contradictory findings with simple additive measures of self-conception correlating more strongly with self-esteem than importance-weighted selfconceptions (Hoge \& McCarthy, 1984).

Pelham and Swann (1989) proposed that the way importance was being measured might account for the weak find- ings in previous studies. These authors suggested it is the intraindividual patterns of differential importance that will affect self-esteem. Similar to the example above regarding the individual who is overweight but highly intelligent, it is the differential importance of these two qualities (intelligence being very important, and body weight being less important) that allows the individual to maintain their selfesteem. Pelham and Swann (1989) examined this differential importance for self esteem. Participants rated themselves on 10 valenced attributes (e.g., intellectual capacity, leadership ability) in relation to other college students (e.g., top 5\%). Participants then completed measures of self-esteem and mood. Results showed that importance did not have a universal effect, such that for individuals with strengths in a number of domains, believing that their greatest strength was important was not necessary to maintain good self-esteem. However, for those participants with relatively few strengths, attributing importance to their greatest strengths had a strong impact on their self-esteem. There was a significant interaction between differential importance and self-view suggesting that importance is only related to the self-esteem of individuals with relatively negative self-conceptions.

Another related variable is that of certainty. How certain a person is about whether or not she or he possesses various attributes may also influence the relationship between selfconcept and self-esteem. Pelham and Swann (1989) found an interaction between differential importance and certainty, suggesting that importance is a predictor of self-esteem only for those who are certain of their more positive selfviews. Therefore, for individuals who only believe they might possess positive attributes, the importance of those attributes does not relate to self-esteem.

There has been some debate in the literature regarding this theory of importance and Marsh (1993, 1995) criticized Pelham and Swann's (1989; see also Pelham, 1995b) findings and suggested that the idiographic ratings of importance may confound individual and group importance ratings. Marsh argued that individual importance ratings will be influenced by society's beliefs about attribute importance. Pelham (1995a) attempted to address these concerns and despite modifications to method and analyses, confirmed the findings from the 1989 study. Pelham (1995a) agreed that societal norms will always influence individual ratings of importance noting that if attaining good self-esteem were as simple as devaluing negative self-aspects, everyone would possess high self-esteem.

Pelham's (1995b) work on importance of self-descriptors used a measure of self-concept that included only 10 discrete attribute domains (e.g., intellectual ability, social skills). The current research examines the self-concept as endorsement of 80 self-referent adjectives, some of which may be applied to more than one domain (e.g., failure, gifted). Using a greater number of descriptive adjectives than in previous research may provide a more comprehensive picture of the role of importance on those descriptors. Further, instead of self-report ratings, reaction time is used as 
a measure of certainty in accordance with the suggestions by Derry and Kuiper (1981).

The present article examines the role of importance ratings of self-descriptors in relation to depressed mood. Schema theory suggests that possessing a depressive schema, a tightly organized network of negative, self-referent information, creates a vulnerability to depression. Other research suggests that the importance individuals place on personal attributes moderates the relationship between self-concept and self-esteem. It is hypothesized that individuals who possess personally important negative attributes will report greater depressive symptoms than those who do not, and individuals who lack personally important positive traits will experience greater depressed mood than those who do not. It is further hypothesized that important self-descriptors will be more strongly related to depressed mood than simply the number of self-referent descriptors. Finally, the relationship between certainty and these variables will be explored.

\section{Method}

\section{Participants}

A posting was placed on the online research participation pool and 115 undergraduate students participated in the study and received course credit for their participation. The mean age of the participants was $19.14(S D=1.42)$ years with a range of $17-27$ and $65 \%$ of the sample was female. The majority of the participants were Caucasian (66\%) and $94 \%$ were in their first year of university. The project was reviewed and approved by the Ethics Review Board of The University of Western Ontario.

\section{Measures}

\section{Beck Depression Inventory-II (BDI-II)}

The BDI-II (Beck, Steer, \& Brown, 1996) is a 21-item selfreport measure. Each item is a multiple-choice question scored on a scale of $0-3$ with higher numbers reflecting increasing severity of depressive symptomatology (Dozois, 2002). Total scores range from 0 to 63 . The BDI-II exhibits excellent validity and reliability in both psychiatric and normal populations (Dozois \& Dobson, 2001; Dozois, Dobson, \& Ahnberg, 1998).

\section{Descriptiveness and Importance Ratings}

Participants were presented with 80 potentially self-descriptive adjectives from a list normed by Meyers (1984) in a self-referent encoding task. Forty words were positive in valence (e.g., admired, trustworthy) and 40 words were negative in valence (e.g., forsaken, failure). Each category was matched on word length, frequency of usage in the English language, and reading difficulty (Meyers, 1984). Each adjective was displayed on the screen and participants indicated "yes" or "no" to the question "Does this word describe you?" This yielded four variables; positive endorsed, positive not endorsed, negative endorsed, negative not endorsed. The computer program recorded length of time to respond. Mean reaction times were computed for the four word-valence combinations. Importance ratings were also collected for each word. For the words with a positive valence, the instructions stated, "Please indicate how important, you feel it is, that you are what the word describes." For the words with a negative valence, the instructions stated, "Please indicate how important, you feel it is, that you are not what the words describes." For both valences, participants indicated their response by the use of a five-point Likert scale, with $0=$ not at all important to $4=$ extremely important. Consequently, four variables were computed: importance of positive words endorsed, importance of positive words not endorsed, importance of negative words endorsed, and importance of negative words not endorsed.

\section{Procedure}

Participants were tested in groups of five or less. To begin, participants were given an informed consent sheet and asked to sign it if they wished to participate. Once the consent form was signed, participants completed the measures on individual computers. The measures were presented in a random order. Upon completion of the tasks, participants were provided with written feedback and given the opportunity to ask any questions they had regarding the study.

\section{Results}

\section{Treatment of the Data and Demographics}

From the data collected, 13 variables were computed. The first was the BDI-II score. The internal consistency $(\alpha)$ value for the scale was .91. The mean for the BDI-II was 11.48 $(S D=8.85)$. This mean is at the 73rd percentile in terms of BDI-II scores in undergraduate samples based on the data from Dozois et al. (1998). BDI-II scores were not significantly correlated with age $(r=-.01, p>.90$; all correlations reported are two-tailed) nor was there a sex difference in scale scores, $t(82)=-1.34, p>.18 .^{1}$

The number of both positive and negative traits endorsed and not endorsed was calculated for each participant. The

\footnotetext{
1 Because of the prevalence of sex differences in depression, analysis of differences in BDI-II scores were examined and reported to ensure that sex effects do not impact the results.
} 
average importance rating for positive and negative words either endorsed or not endorsed was also computed. Independent sample $t$-tests demonstrated no significant sex differences on these variables. Average reaction time data was computed for positive and negative traits, separately for both endorsed and not endorsed. For these variables, men were found to have slightly higher (slower) average reaction time scores for positive endorsed traits, 1308.90 vs. $1130.39, t(82)=2.26, p<.05$. No other comparisons between men and women were significant.

\section{Assessing Agreement in Importance Ratings}

To address Marsh's (1993, 1995) concern that societal norms would influence importance ratings, analyses examined whether or not participants were in high agreement in their ratings of the importance of each item by analyzing the variance in importance ratings. The positive and negative terms were examined separately. In particular, the variance values for each item were computed and the means and standard deviations of the variance values were computed. If participants were in complete agreement in their ratings of the importance of the items, then the variance values would be close to zero. The mean variance values for the positive and negative terms were compared using a single-sample $t$-test against a theoretical mean of zero. For both positive, $(t(39)=25.43, p<.001$, and negative, $t(39)$ $=23.58, p<.001$, ]items, the average variance scores were found to significantly differ from zero, suggesting that the participants were not in agreement in their ratings of the importance of the items and that individual differences exist in how people rated the importance of the items.

\section{BDI-II Scores and Importance of Attributes}

The relationship between depressed mood and endorsement rates was examined. The total number of positive words endorsed was found to have a strong negative correlation with BDI-II scores $(r=-.45, p<.001)$ suggesting that greater depressed mood is related to lower endorsement of positive traits. Similarly, the total number of endorsed negative words was found to have a strong significant positive correlation $(r$ $=.58, p<.001)$ suggesting that greater depressed mood is related to higher endorsement of negative traits. Because the number of items not endorsed is simply the number of items endorsed subtracted from the total number of items, the correlations with the nonendorsed items and the BDI-II scores are the same values in reverse order (i.e., $r=.45$ between number of positive items not endorsed and $r=-.58$ between number of negative items not endorsed).

The average importance ratings for endorsed negative and positive traits were computed separately. For both the positive $(r=.16, p>.10)$ and negative descriptors $(r=.02, p>$ $.80)$, the average importance rating did not correlate significantly with BDI-II scores. It appears that the importance of traits that individuals possess is not related to mood. The average importance values for the items not endorsed, however, did correlate significantly with BDI-II scores. The average importance rating of positive terms not endorsed correlated $.43(p<.001)$ with BDI-II scores, suggesting that the greater the importance of positive traits that a person lacks, the higher the depressed mood. The correlation between the average importance ratings of negative items which were not endorsed was negatively correlated with BDI-II scores ( $r=$ $-.35, p<.001)$, suggesting that the more important the negative traits the person is free of, the lower the depressed mood.

\section{BDI-II Scores and Reaction Times}

To examine the relationship between BDI-II scores and the speed with which individuals endorsed the descriptors, average reaction time scores were computed separately for endorsed positive items, endorsed negative items, not endorsed positive items, and not endorsed negative items (see Table 1). The results suggest that those who score higher on the BDI-II tend to be more certain about positive traits endorsed and less certain about negative traits endorsed and positive traits not endorsed. A nonsignificant negative correlation was found between negative terms that were not endorsed and BDI-II scores $(r=-.03, p>.10)$, indicating that depressed mood was not related to the certainty of not endorsing negative traits.

Table 1. Correlations between reaction times and BDI-II scores

\begin{tabular}{ll}
\hline & BDI-II Scores \\
\hline Endorsed & \\
Positive & $-.24 *$ \\
Negative & $.56^{* *}$ \\
Not endorsed & \\
Positive & $.32 * *$ \\
Negative & -.03 \\
\hline
\end{tabular}

$* p<.05 ; * * p<.01 ;$ two-tailed

Note: longer reaction times imply less certainty in decision making

\section{Reaction Times and Importance Ratings}

Reaction times and importance ratings were computed for the four category pairs: positive words endorsed, negative words endorsed, positive words not endorsed, and negative words not endorsed. The only significant relationship was found between importance ratings and reaction times was between the importance ratings and reaction times of positive items not endorsed $(r=.79, p<.01)$. This positive correlation suggests people took longer when responding to important positive terms which they decided were not self-descriptive. Based on these results, the importance of a trait does not consistently relate to the certainty of endorsement. 


\section{Index of Differential Certainty and Importance}

Following the methodology employed by Pelhman and Swann (1989), a within-subject differential index was computed. In particular, for positive and negative terms separately, the correlation between the participant's rating of the importance of an item with their decision (reaction) time was computed. A positive correlation suggested that a person took longer to decide on items that they deemed to be important and were, therefore, less confident. A negative correlation suggested that the person was more confident as they were faster to respond to more important items. These within-subject correlations were then correlated with the BDI-II total scores. The correlation between the certainty-importance index and BDI-II for positive terms was .03 (ns), suggesting that there was no relationship between certainty-importance for positive terms and mood scores. In contrast, the correlation between the certainty-importance index values and the BDI for negative terms was -.23 $(p<.05$, two-tailed). This correlation suggests that people experiencing greater depressed mood were more certain about endorsing negative traits they deemed important.

\section{Discussion}

Marsh (1993, 1995) suggested that society's norms and values would influence idiographic ratings of importance of attributes, creating homogeneity in ratings of importance and, thereby, rendering this investigation pointless. However, the current results suggest that the variance in respondents' importance ratings was great enough to indicate that individuals did indeed rate attribute importance differently. So, while society's members may be in agreement to a certain degree, there are individual differences in how important it is to possess or not possess certain characteristics.

As expected, depressed mood was related to the endorsement of adjectives with participants who experienced depressed mood endorsing more negative and fewer positive characteristics. This is consistent with previous research examining the content of negative self-schemas in depressed individuals (Clark et al., 1999; Derry \& Kuiper, 1981). It was hypothesized that the importance that a person placed on a self-descriptor would influence the relationship between mood and endorsement of that descriptor. However, importance ratings did not do so consistently. Importance did not matter for words that were endorsed as self-descriptive, but it did matter for words that were not endorsed. This finding suggests that the importance of selfattributes may be less salient for traits that a person possesses than for those he or she perceives as absent. In effect, lacking important positive characteristics was related to greater depressed mood, whereas being free of important negative characteristics was related to lower depressed mood.

This finding would suggest that the importance of positive attributes that one possesses is not related to one's mood. This may be related to what Pelham and Swann (1989) found with the importance of the individuals' greatest strength not being necessary for self-esteem. Further, possessing negative characteristics that an individual feels are important to be free of was also not related to depressed mood. This also hints at the possibility for the intraindividual differential index that Pelham and Swann (1989) suggested. Simply possessing positive attributes is not enough to prevent dysphoric mood. One must also not be lacking in important positive characteristics. Further, possessing negative characteristics does not directly lead to greater depressed mood, but being free of important negative traits may help to reduce depressed mood.

An examination of the reaction times revealed interesting findings. Recall that reaction times were used as a measure of certainty on the assumption that faster endorsement meant the individual did not need to deliberate on the item. Individuals who reported greater depressed mood were: (1) more certain of positive characteristics they possessed, (2) less certain of negative characteristics they possessed, and (3) less certain of positive characteristics they lacked. There was no relationship between mood and the reaction times for negative characteristics possessed. These findings are somewhat unexpected based on the schema theory. The schema theory proposes a depressed individual should be very aware of the negative characteristics they possess because this information would be schema congruent. Also, depressed individuals should be less certain of the positive characteristics they possess because that information should be schema incongruent. These findings appear to be in disagreement with the results found by Derry and Kuiper (1981), which showed no difference in reaction times between depressed and nondepressed controls regardless of positive or negative content.

However, when examining reaction times (i.e., certainty) in conjunction with importance ratings we see a somewhat different picture. While higher BDI-II scores are related to slower reaction times for endorsing negative adjectives in general, they are related to faster times for endorsing negative adjectives that the respondent sees as important. This is an interesting finding because it suggests that not all negative information is processed equally, and that the value the individual places on the information may be having an impact. It is not just negative information that is processed differently for those individuals with a depressive schema, but, specifically, value-laden negative information.

The current findings need to be replicated before making any claims and a great deal more research is needed in this area before any conclusions can be drawn, but this research suggests that things may not be exactly as expected. The importance of the characteristics individuals possess is influencing the relationship between mood and self-concept 
in a way that was not anticipated based on the schema theory of depression. Research now needs to investigate this process in greater depth to ascertain how the characteristics and their importance/value relate to each other in clinically depressed and nondepressed individuals. Is it a matter of balance between characteristics possessed and lacked and the value that each individual assigns to them that protects some individuals from experiencing depression? Research examining individuals and their own intraindividual patterns of differential importance is needed in order to understand this balance.

\section{References}

Beck, A.T. (1967). Depression, clinical, experimental, and theoretical aspects. New York: Harper \& Row.

Beck, A.T., Steer, R.A., \& Brown, G.K. (1996). Beck Depression Inventory manual (2nd ed.). San Antonio, TX: Psychological Corporation.

Clark, D.A., Beck, A.T., \& Alford, B.A. (1999). Scientific foundations of cognitive theory and therapy of depression. New York: Wiley.

Derry, P.A., \& Kuiper, N.A. (1981). Schematic processing and self-reference in clinical depression. Journal of Abnormal Psychology, 90, 286-297.

Dozois, D.J.A. (2002). Cognitive organization of self-schematic content in nondysphoric, mildly dysphoric, and moderately-severely dysphoric individuals. Cognitive Therapy and Research, 26, 417-429.

Dozois, D.J.A., \& Dobson, K.S. (2001). A longitudinal investigation of information processing and cognitive organization in clinical depression: Stability of schematic interconnectedness. Journal of Consulting and Clinical Psychology, 69, 914-925.

Dozois, D.J.A., Dobson, K.S., \& Ahnberg, J.L. (1998). A psychometric evaluation of the Beck Depression Inventory-II. Psychological Assessment, 10, 83-89.

Gotlib, I.H., \& Krasnoperova, E. (1998). Biased information processing as a vulnerability factor for depression. Behavior Therapy, 29, 603-617.

Hoge, D.R., \& McCarthy, J.D. (1984). Influence of individual and group identity salience in the global self-esteem of youth. Journal of Personality and Social Psychology, 47, 403-414.

Ingram, R.E. (1984). Toward an information-processing analysis of depression. Cognitive Therapy and Research, 8, 443-478.

James, W. (1890). The principles of psychology. New York: Holt.
Kaplan, H.B. (1980). Deviant behavior in defence of self. San Diego: Academic Press.

Marsh, H.W. (1986). Global self-esteem: Its relation to specific facets of self-concept and their importance. Journal of Personality and Social Psychology, 51, 1224-1236.

Marsh, H.W. (1993). Relations between global and specific domains of self: The importance of individual importance, certainty, and ideals. Journal of Personality and Social Psychology, 65, 975-992.

Marsh, H.W. (1995). A Jamesian model of self-investment and self-esteem: Comment on Pelham (1995). Journal of Personality and Social Psychology, 69, 1151-1160.

Meyers, J. (1984). Schematic processing and self-reference during continuous periods of clinical depression and symptomatic remission. Unpublished doctoral dissertation, University of Calgary, Calgary, Alberta.

Pelham, B.W. (1995a). Further evidence of a Jamesian model of self-worth: A reply to Marsh (1995). Journal of Personality and Social Psychology, 69, 1161-1165.

Pelham, B.W. (1995b). Self-investment and self-esteem: Evidence for a Jamesian model of self-worth. Journal of Personality and Social Psychology, 69, 1141-1150.

Pelham, B.W., \& Swann, W.B. (1989). From self-conceptions to self-worth: On the sources and structure of global self-esteem. Journal of Personality and Social Psychology, 57, 672-680.

Rosenberg, M. (1965). Society and the adolescent self-image. Princeton: Princeton University Press.

Rosenberg, M. (1979). Conceiving the self. New York: Basic Books.

Segal, Z.V. (1988). Appraisal of the self-schema concept in cognitive models of depression. Psychological Bulletin, 103, $147-162$.

Segal, Z.V., \& Gemar, M. (1997). Changes in cognitive organization for negative self-referent material following cognitive behavioral therapy for depression: A primed Stroop study. Cognition and Emotion, 11, 501-516.

\section{J.K. Brinker}

Department of Psychology

The University of Western Ontario

Social Science Center

London, Ontario

Canada N6A 5C2

E-mail jbrinker@uwo.ca 